\title{
FACTORES POTENCIALES QUE INFLUYEN EN EL ABANDONO ESCOLAR DE LOS ESTUDIANTES DE LA ESCUELA TELESECUNDARIA 531 FRIDA KAHLO
}

POTENTIAL FACTORS INFLUENCING THE SCHOOL DROP-OUT OF STUDENTS AT THE 531 FRIDA KAHLO REMOTE SCHOOL

Gerbin Didier Rodas Vera dirog_013@hotmail.com 
Para citar este artículo:

Rodas Vera, G. D. (2021). Factores potenciales que influyen en el abandono escolar de los estudiantes de la Escuela telesecundaria 531 Frida Kahlo. ESPACIO I+D, INNOVACIÓN MÁS DESARROLLO, 10(26). https://doi.org/10.31644/IMASD.26.2021.a06

\section{RESUMEN}

La incorporación obligatoria, en México, a la educación básica, se ha considerado un gran avance en materia educativa, aunque el esfuerzo realizado ha implicado resolver una serie de retos, como el de la permanencia en las escuelas. Bajo diversas modalidades se ha pretendido la atención a la cobertura en la educación secundaria, como es el caso de esta investigación sobre el abandono escolar en educación telesecundaria en contextos indígenas en Chiapas, lo cual se constituye en un acercamiento para comprender la dinámica que se vive en estos escenarios. Su objetivo es identificar los factores potenciales que pueden incidir en el abandono escolar de los estudiantes de la escuela telesecundaria 531. El estudio de caso se utilizó para encontrar las causas mediante una serie de instrumentos con los que se recabó información por parte de los diversos autores que forman parte del contexto. En este caso los participantes fueron 40 estudiantes de tercer grado, 20 padres de familia y 5 profesores.

Se encontraron como principales factores potenciales: la influencia de la familia en torno al cumplimiento de los usos y costumbres relacionadas con contraer matrimonio a edades tempranas, un fuerte patriarcado, con respecto a la concepción de la mujer y la violencia de género en la determinación de lo que debe hacer con su vida futura, un escaso interés por sus estudios y la falta de recursos económicos para continuar en la escuela. La existencia de estos factores requiere una seria reflexión por parte del profesorado y las autoridades educativas para el planteamiento de estrategias que permitan una permanencia y egreso con calidad en el contexto de la educación indígena. Asimismo, es importante realizar estudios comparativos con otras realidades similares a ésta para comprender a fondo los problemas relacionados con la equidad en la educación.

\section{Palabras clave}

Abandono escolar; factores potenciales; deserción escolar; educación; educación indígena. 
The compulsory incorporation in basic education in Mexico has been considered a great advance in educational matters, although the effort made has implied solving a series of challenges, such as the permanence in schools. Under various modalities, attention to coverage in secondary education has been sought, as is the case of this research on dropout in telesecundaria education in indigenous contexts in Chiapas, which constitutes an approach to understand the dynamics that are lived in these scenarios. Its objective is to identify the potential factors that may influence the drop-out of students in telesecundaria 531. The case study was used to find the causes through a series of instruments that collected information from various authors who are part of the context. In this case, the participants were 40 third grade students, 20 parents and 5 teachers.

The main potential factors were the influence of the family around the fulfillment of the uses and customs related to marrying at an early age, a strong patriarchy with respect to the conception of women and gender violence in determining what he must do with his future life, a low interest in his studies and the lack of financial resources to continue in school. The existence of these factors requires a serious reflection on the part of the teaching staff and the educational authorities for the planning of strategies that allow a permanence and quality graduation in this context of indigenous education. Likewise, it is important to carry out comparative studies with other realities similar to this one to fully understand the problems related to equity in education.

\section{Keywords}

School drop-out; potential factors; dropout; education; indigenous education. 
bordar la educación secundaria en los pueblos originarios es atender
la complejidad de una realidad que es distinta en muchos aspectos
a lo que habitualmente se conoce desde la visión occidentalizada. Hemos sido testigos de la presencia de proyectos asimilacionistas, caracterizados por la imposición de un modelo educativo hegemónico que no atiende la cultura ni la diversidad cultural de estos pueblos. De allí que, a últimas fechas, se hable de la introducción, en lo educativo, de un enfoque basado en la interculturalidad.

En el caso de la educación telesecundaria, esta se ofrece en las comunidades con alta marginación y se pretende que, a través de ésta, exista un cambio en la forma de pensar sobre los procesos educativos. Sin embargo, se reconoce que existen una serie de problemas, desde los relacionados con el bilingüismo y la poca preparación de los profesores, hasta el acceso muy limitado a la conectividad y el abandono escolar.

Se ha reconocido que el fenómeno histórico de abandonar la escuela tiene múltiples factores, por lo que resulta importante conocer las causas tanto en los egresados como en los que todavía se encuentran en las aulas. A estos últimos se les conocerá como factores potenciales. En esta situación, la escuela telesecundaria 531 Frida Kahlo presenta un alto índice de abandono escolar detectado en los últimos tres ciclos escolares, existe un mayor porcentaje de abandono en las aulas, como se describe más adelante, por lo que se presentarán los resultados de la investigación que tuvo como objetivo identificar los factores potenciales que pueden incidir en el abandono escolar de los estudiantes de la escuela telesecundaria 531.

Se aborda esta problemática a partir de antecedentes teóricos que fundamentan el estudio realizado por diferentes autores, quienes investigan los factores de abandono escolar y la interculturalidad que está presente en gran parte de las instituciones del país, tal es el caso de la Telesecundaria 531, que se ubica en una zona rural de la lengua indígena tzotzil, con una forma de gobierno de acuerdo con usos y costumbres.

Con respecto al uso de los resultados de esta investigación en su impacto social, se espera que se pueda atender con calidad las expectativas y necesidades de la educación indígena en un contexto de interculturalidad. También conlleva a repensar las estrategias de atención a la cobertura, que siempre ha sido de forma homogénea sin tomar en consideración el valor que las comunidades indígenas le dan a las prácticas de vida cotidiana por sobre la educación de carácter obligatorio, como lo es la secundaria.

Por otra parte, a diferencia de los clásicos estudios sobre abandono escolar que ponen su atención en los estudiantes que no lograron concluir sus estudios, esta investigación se caracteriza por ser un ejercicio en el que se coloca al estudiante que todavía está en los espacios escolares y retoma 
su forma de percibir su proceso formativo, contribuyendo con esto al campo disciplinario, desde un planteamiento metodológico en el que los sujetos de investigación todavía se encuentran en las aulas. Sin embargo, en cuanto a que el trabajo tiende más a un enfoque cualitativo y ayuda a la comprensión de la problemática, por lo que se espera se lleven a cabo estudios comparativos con otras realidades similares a ésta, para comprender a fondo los problemas relacionados con la equidad en la educación.

Para llevar a cabo el trabajo de investigación se considera pertinente el estudio de caso, con una muestra de docentes, alumnos y padres de familia, utilizando como instrumentos de investigación encuestas, por medio de cuestionarios, a los estudiantes, así como entrevistas colectivas a padres de familia y profesores, para recabar información referente a cómo influye la cultura de los padres, la situación económica y la comprensión de los contenidos en el abandono escolar. Es importante conocer los factores que lo generan, para crear una educación inclusiva, integral e intercultural en los estudiantes.

\section{DESARROLLO}

El abandono escolar es un problema educativo que tiene muchas repercusiones tanto a nivel personal como a nivel social. Una de estas consecuencias negativas tiene que ver con el riesgo de contar en el futuro con recursos humanos de baja calidad. En esta investigación se busca conocer los motivos de esta problemática al llevar a cabo la revisión del concepto de abandono escolar desde diversas miradas teóricas, al tiempo de identificar la forma en que se ha estudiado esta problemática y los factores que han encontrado y que inciden de acuerdo con los autores que investigan sobre el tema.

Zúñiga (2006), Navarro (2001), Espíndola y León (2002), entre otros han estudiado la problemática del abandono escolar en diferentes niveles educativos, coincidiendo en la conceptualización de la acción de abandonar los estudios en cualquier nivel educativo, ya sea de manera definitiva o temporal. Esto se señala porque la decisión de ingresar o no nuevamente al sistema educativo dependerá de muchas condiciones, entre ellas las necesidades de escolarización que requieran los puestos de trabajo, la facilidad para incorporarse a los estudios, el apoyo personal o familiar, entre otros.

Estrada (2018) coordina un texto sobre el abandono escolar en la educación media superior de México, centrando su atención en las políticas y actores desde el análisis de caso. En su texto, organizado en tres partes, reúne una serie de capítulos para analizar el fenómeno desde las políticas, programas e instituciones, así como desde los directivos, docentes, padres de familia y jóvenes. Ibarra (2018) desde el paradigma de investigación-acción se pregunta ¿por qué se van de la escuela? señalando que, aunque en su mayoría los jóvenes indican que lo hacen por motivos personales, existen otras razones 
como por ejemplo las de tipo curricular, los directivos y hasta los docentes, los cuales pueden estar generando riesgo de exclusión.

Con respecto a los factores o causas, Espíndola y León (2002) señalan que pueden provenir de factores extraescolares relativos a las condiciones personales y contextuales del alumno y de otros que pueden estar asociadas a las insuficiencias del sistema educativo a los que denominó intraescolares. Consideran que las causas del abandono escolar pueden clasificarse en: a) razones económicas, b) problemas relacionados con la oferta educativa en la región, c) problemas familiares relacionados con la forma de organización de la vida doméstica, d) falta de interés, e) bajo rendimiento y f) problemas de conducta.

No puede olvidarse que el contexto es un elemento que determina en gran medida el comportamiento del estudiantado, sobre todo cuando existe poca coincidencia con las prácticas culturales y las dinámicas institucionales de los planteles educativos. Por ejemplo, el uso del uniforme, la asistencia diaria o el cumplimiento en tareas, no resultan comprendidas de la misma forma en una población vulnerable y en comunidades alejadas de todos los servicios, a una donde tales servicios están garantizados.

Cabe mencionar que estas causas no pueden presentarse de forma única sino una combinación de ellas. Por ejemplo, el embarazo no deseado puede dar lugar a problemas de rendimiento, problemas familiares, de comportamiento, entre otros. De ahí la complejidad para entender el problema del abandono, en cada nivel educativo.

Leiva (2013), sugiere, debido a las causas, que cuando se atienden las necesidades y se responde a la diversidad de características de los estudiantes, se puede reducir la exclusión en los sistemas educativos, puesto que se logra identificar el posible origen de la problemática, siempre y cuando se atienda a tiempo y de manera eficaz. Tal puede ser el caso de la respuesta a esta problemática con una visión occidentalizada que no comprende la cultura y el valor de la educación en un contexto con culturas originarias y que pretende establecer rutinas y rituales escolares que son ajenos al contexto. Un ejemplo de ello puede ser el de las escuelas rurales indígenas que chocan con un currículum homogéneo, generando condiciones que pueden tener como salida el abandono escolar, tal como lo señala el Instituto Nacional de Lenguas Indígenas, INALI (2008, p.39) destacando que:

Actualmente los planes de estudio de educación básica son los mismos a nivel nacional, con escasas variantes para adaptarlos a las necesidades específicas de la población a la que va dirigido; asimismo, persiste el criterio homogeneizador que no contempla el respeto a la diversidad, a la pluralidad cultural y al multilingüismo. Vinculado con lo anterior, se observa un desarrollo insuficiente de materiales didácticos en lenguas indígenas con enfoque intercultural y multilingüe. 
Esto es algo sobre lo que deben reflexionar los planeadores y administradores educativos cuando en el cumplimiento de la atención a la cobertura tienen una sola mirada, la occidentalizada, para educar a todos de la misma manera. No tomar en cuenta las características y necesidades de los estudiantes es una situación que podría poner en riesgo y provocar exclusión del sistema educativo, pese a las buenas intenciones. El problema de la exclusión escolar ha sido ampliamente estudiado desde diversas perspectivas teóricas, tal es el caso de los sociólogos de la educación, quienes conciben a la escuela como un espacio al que no todos pueden acceder, ya sea por su código lingüístico restringido (Bernstein, 1975, como se citó en Usategui, 1992) o por un pobre capital cultural (Bourdieu y Passeron, 1981).

El abandono escolar ha sido estudiado también por Sandoval (2018) quien indaga sobre los factores que inciden en la deserción escolar en los estudiantes de educación básica secundaria y media superior de la escuela Técnica Félix Tiberio Guzmán, de El Espinal Tolima, utilizando como método el estudio de caso. Encontró que los motivos más comunes a la hora de cancelar la matrícula fueron el cambio de domicilio o cambio de ciudad, pues la situación económica de las familias cada día es más difícil y en especial con la importación de arroz y el trabajo informal en aumento.

Se encontró también que el trabajo infantil es muy común en las familias de escasos recursos económicos y muchas de éstas son disfuncionales, por lo que los jóvenes deben ayudar trabajando para el sustento familiar. De acuerdo con Sandoval (2018) en sus resultados observó que la mayor deserción se da en la educación básica secundaria con 38 estudiantes. También encontró que la deserción en educación primaria fue de 24 estudiantes. Un factor que identificó en su estudio se relaciona con la apatía hacia la escolarización de los estudiantes, quienes manifestaron que no les agradaba la forma en que los docentes respondían a sus dudas y preferían quedarse callados, generando con ello problemas conceptuales, lo que por ende se reflejaba en un pobre rendimiento académico.

En el caso de González, et al. (2016, p.8) se investigaron los factores sociales que influyen en el abandono escolar temprano, a través de la revisión y análisis se dedicaron a explorar el problema en educación obligatoria. Los resultados que encontraron en esta revisión, que abarca un periodo de estudios sobre el abandono de los últimos cien años, permite concluir que:

Los factores que influyen en el abandono escolar temprano responden a un modelo complejo, en el que las causas no son independientes, sino que interactúan entre sí. Un ejemplo de ello lo encontramos en los bajos ingresos, la pérdida de empleo, el estrés familiar o la educación de los padres, ya que todos ellos tienen una relación directa con el abandono escolar temprano. Por tanto, para un correcto diagnóstico a partir del cual se puedan proponer soluciones 
es necesario que el entorno familia-escuela tenga una visión global de esta complejidad, ya que resulta fundamental poder ver el problema desde todas las perspectivas posibles.

Hernández, et al. (2019) desarrolló una investigación para conocer los factores de abandono escolar del iево 40 San Mateo del Mar en Oaxaca, encontrando que la mayoría de los estudiantes de dicho plantel manifestaron que el factor socioeconómico es el más importante que los obliga a abandonar sus estudios. Otro hecho resulta ser el factor familiar (desintegración) que interviene en el abandono escolar, donde no existe una buena relación entre integrantes de la familia, donde hay un mayor grado de ambiente desagradable. Si no hay apoyo entre los integrantes de la familia, o cuando es una familia disfuncional, puede ocasionar que el estudiante deserte en sus estudios.

En la misma investigación se identificó que hay estudiantes que manifiestan la falta de la figura paterna y por lo general los hijos varones son los que tienen que dejar la escuela para poder trabajar y apoyar en el sustento del hogar. Otro aspecto importante se relaciona con la forma en que los estudiantes se valoran y perciben, adicionalmente pueden tener pocas estrategias para afrontar las tareas académicas o personales e incluso algunos pueden tener problemas relacionados con su personalidad.

Hernández, et al.(2019), identifica como otra causa la falta de claridad en las metas que se proponen los estudiantes, lo cual no les permite definir hacia dónde deben dirigirse o cómo deben hacerlo para lograr lo que se proponen. Los investigadores sugieren la necesidad institucional de diseñar una ficha de control por cada estudiante, para darle seguimiento a su comportamiento que incluya lo siguiente: a) motivos de deserción, b) escolaridad de los padres, c) apoyos económicos de la escuela, d) tipo de trabajo de los padres; de igual manera proponen un modelo econométrico que mida las probabilidades de deserción dado los factores que anteriormente se comentaron y finalmente elaborar un programa de apoyo.

González (2016) investiga la motivación y abandono escolar en educación media superior con el objetivo principal de ampliar la comprensión de los factores asociados a esta problemática desde los aportes de la psicología de la motivación. En sus resultados se pudo evidenciar la influencia que ejercen los factores de tipo endógenos en el fenómeno del abandono escolar, así como en la permanencia de los jóvenes en el sistema educativo. Se destaca entre estos el desencuentro entre las expectativas de los jóvenes con la rigidez de los planes curriculares y los contenidos académicos, los climas de clase caracterizados por el control y las presiones extrínsecas, y las relaciones negativas que tienen los estudiantes con sus docentes y sus pares. También, encontraron que se percibe la existencia de una insatisfacción por parte de los estudiantes, en el cumplimiento de sus expectativas y necesidades 
con respecto a las instituciones educativas. En algunos casos otro factor relacionado fue el alejamiento de los centros educativos en Educación Media y la falta de adaptación de los estudiantes con respecto al contenido enseñado adicionalmente a vínculos poco satisfactorios con profesores y pares, lo que les provocaba una gran desmotivación.

Como un factor que puede incidir en el abandono escolar, Leiva (2017), estudia el fenómeno de la interculturalidad como valor educativo y también como praxis para la mejora de la inclusión social y emocional de todos los agentes de la comunidad educativa. En este sentido, se ha considerado la importancia de identificar el valor de las diferencias individuales en las prácticas pedagógicas. Señala que se ha minimizado lo cultural en el factor pedagógico con equipos docentes que no contemplan la diversidad cultural como un elemento de interés y relevancia en lo educativo, trayendo como consecuencia que se ahonden los estereotipos sobre alumnos y familias de orígenes culturales diversos.

Cuando se parte del principio de interculturalidad en las aulas, éste debe servir para promover el componente relacional y emocional de los aprendizajes y los procesos de interiorización crítica sobre las preguntas clave de la educación de todos los tiempos: el qué, el para qué, el cómo, el por qué, el cuándo, el dónde para dar sentido a lo educativo. Reconocer el valor de las diferencias individuales a partir de los rasgos culturales de cada estudiante es un factor importante para aprender de cada uno en el espacio áulico y que se pueda co-construir el conocimiento, dando valor a los saberes de cada uno.

\section{La educación telesecundaria en Chiapas}

Núñez y Jiménez (2016) encontraron que, de las tres modalidades de servicio, que ofrecen educación secundaria, la telesecundaria ha experimentado el mayor crecimiento en los últimos años, dada la facilidad del sistema para implementarse en zonas rurales. En el caso de Chiapas, estos autores señalan que más del $46 \%$ de la matrícula en secundaria es atendida en esta modalidad. Sin embargo, argumentan que si bien las telesecundarias han permitido este sustantivo incremento de la cobertura, no son una respuesta adecuada para promover la continuidad en el Sistema Educativo, puesto que reconocen que existe una alta tasa de deserción. En muchos casos la secundaria es el último nivel educativo que cursan y que repercuten en el reducido ingreso al nivel medio superior.

Según datos del Instituto Nacional de Lenguas Indígenas (INALI, 2008), en Chiapas el abandono escolar en primaria con cifras al 2013 alcanzó una tasa del $1.3 \%$. Esta cifra se identifica que fue más del doble de las cifras nacionales, lo cual es preocupante por las consecuencias negativas en el 
desarrollo tanto personal como del estado. Entre las causas del abandono se menciona que el $91.4 \%$ lo hizo porque realiza otra actividad, ya sea porque se insertó en el mercado laboral en un $64.1 \%$ o se dedicó a las labores del hogar en un $24 \%$, según lo indica este estudio.

Por otra parte, el reconocimiento constitucional de México como país pluricultural en 1992, puso sobre la mesa una serie de problemas por atender con respecto a la desigualdad en aspectos económicos, políticos, sociales y culturales, el cual es un asunto pendiente para los pueblos indígenas. Desde 1993 tanto el sistema educativo nacional como el estado mexicano desarrollaron estrategias para favorecer la equidad y el derecho humano de calidad. Esto se expresa en la Ley General de Educación, artículo 7, apartado IV, que ratifica: "Promover mediante la enseñanza el conocimiento de la pluralidad lingüística de la Nación y el respeto a los derechos lingüísticos de los pueblos indígenas. Los hablantes de lenguas indígenas tendrán acceso a la educación obligatoria en su propia lengua y español" (1993, p.2).

En Chiapas, oficialmente se reconocen once lenguas: Tzeltal, Tzotzil, Chol, Zoque, Tojolabal, Mam, Q'anjob’al, Chuj, Akateco, Zapoteco, Lacandón; siendo el más numeroso el Tzeltal, con $39.5 \%$ del total de hablantes y el menos el lacandón con el 0.1 \% (INEGI, 2015). Sin embargo, existen muchas variantes de estas lenguas, lo que dificulta en gran medida hacer efectivo ese derecho a la educación, que implica contar con maestros preparados que hablen lenguas indígenas.

\section{METODOLOGÍA}

La investigación tuvo como objetivo general identificar los factores potenciales que pueden incidir en el abandono escolar de los estudiantes de la Escuela Telesecundaria 531 Frida Kahlo en el ciclo escolar 2019-2020.

El método utilizado para la investigación es el estudio de caso, el cual se entiende como un método caracterizado por conocer y comprender la particularidad de una situación para identificar su funcionamiento. Esta metodología de estudio de caso es una técnica de investigación cualitativa, puesto que el desarrollo se centra en el estudio de un fenómeno no para generalizar sus resultados, sino para analizar la complejidad del objeto de estudio, desde todos los ángulos posibles y la mayoría de los implicados en el mismo.

En palabras de Díaz, et al. (2011, p.21) "la esencia del estudio de caso es la descripción, explicación o comprensión de un inter/sujeto/objeto, una institución, un entorno o una situación única y de una manera lo más intensa y detallada posible”.

Esto permite indagar a profundidad un problema objeto de estudio a través de diversos instrumentos como los utilizados en esta investigación: las entrevistas realizadas a los padres de familia y a los docentes, así como 
encuestas a los estudiantes, con la finalidad de identificar ¿Cuáles son los factores potenciales que pueden incidir en el abandono escolar de los alumnos de la escuela telesecundaria 531 ?

Tal y como señala Ceballos-Herrera (2009), la investigación basada en el estudio de caso pertenece al paradigma cualitativo interpretativo en tanto que la realidad es construida por las personas que están involucradas en la situación que se estudia.

Tomando en cuenta lo que sugiere Morse y Rojas (2001), citado por Hernández, Fernández y Baptista (2018), es necesario establecer los límites espaciales y temporales de la investigación, en este estudio hace referencia a los estudiantes de ambos géneros de la Escuela Telesecundaria 531 "Frida Kahlo", ubicada en la comunidad Carmen Zacatal, perteneciente al Municipio de Jitotol, Chiapas. Respecto a la delimitación temporal, esta investigación cubre el periodo comprendido de agosto del 2019 a junio del 2020, un ciclo escolar para la observación de los factores que influyen en el abandono escolar, como ya se dijo antes en el contexto de la Escuela Telesecundaria 531.

La investigación se llevó a cabo con una muestra de 5 docentes que laboran frente a grupo en la institución, que representan el $55.5 \%$, tres docentes del sexo femenino y dos del sexo masculino, con una antigüedad laboral de 17 años de servicio en la docencia, en una edad aproximada de 40 años. Tres maestras atienden los tres grupos de tercer grado y los dos docentes segundo grado.

La muestra estuvo constituida por un total de 40 estudiantes que, al momento del estudio, se encontraban cursando el tercer grado y que accedieron de manera voluntaria a contestar el instrumento por lo que el muestreo fue de tipo deliberado, se eligió a los estudiantes del tercer grado por estar cursando el último año de educación básica, con una edad aproximada de 15 años, es el grado escolar en que se puede apreciar las deserciones ocurridas desde su ingreso a la telesecundaria en el año 2017 a la fecha, de los cuales el $60 \%$ pertenecen al sexo femenino y el $40 \%$ pertenecen al masculino.

La muestra de los padres de familia estuvo constituida por 20 padres de familia, con edades entre 30 y 35 años cuya ocupación es labores del campo en la agricultura, se dedican al cultivo de maíz y café, son de origen indígena tzotzil.

La Telesecundaria es un servicio de educación básica, público y escolarizado que se dirige a la educación de jóvenes de zonas rurales e indígenas, así como urbano-marginadas. Además, toma como referencia los índices de marginación establecidos por el Consejo Nacional de Población (CONAPO, 2015), relacionados con la falta de acceso a la educación, viviendas inadecuadas, ingresos monetarios insuficientes y localidades pequeñas con menos de 2,500 habitantes. 
En el caso del estudio presentado, la escuela Telesecundaria Frida Kahlo, cuenta con una planta docente de 9 profesores a cargo de 9 grupos y un director. De acuerdo con los datos proporcionados por su director, la deserción escolar en el ciclo escolar 2017-2018 fue de 40 estudiantes, lo que representa un $14 \%$ del total de la población escolar. En el ciclo escolar 2018-2019 se presentaron 39 bajas de alumnos de un total de 279 alumnos inscritos al inicio del ciclo escolar, 22 son hombres y 17 mujeres. Se presenta un $13.97 \%$ de deserción.

Los datos en el sistema de administración educativa de Chiapas indican que al inicio del ciclo escolar 2019-2020, de acuerdo con los registros de los alumnos que terminaron el primero y segundo grado de secundaria, fueron 171 alumnos, de los cuales se inscribieron únicamente 134 alumnos, desertaron 37 alumnos lo que representa $21.63 \%$, más 30 deserciones al mes de marzo del presente año, como puede apreciarse hay un alto índice de deserción escolar en esta Institución educativa.

Se utilizó como instrumento de recolección de datos un cuestionario con 5 preguntas abiertas y 5 preguntas cerradas a los estudiantes. Entrevista de 5 preguntas abiertas a los docentes de la institución, entrevista de 5 preguntas abiertas a los padres de familia para explorar los siguientes factores: a) Usos y costumbres; b) Violencia de género; c) Situación económica; d) Comprensión de los contenidos escolares; e) Estrategias didácticas. Estas categorías se fueron identificando a lo largo del ciclo escolar, en las pláticas informales con estudiantes y profesores de la escuela telesecundaria, las cuales sirvieron como referente para ser utilizadas en los instrumentos.

Las entrevistas y el cuestionario se aplicaron durante cuatro meses, del 12 de noviembre al 12 de marzo del ciclo escolar 2019-2020, en las instalaciones de la institución con alumnos y docentes, con los padres de familia en reuniones formales. Se llevó a cabo la codificación de las preguntas abiertas para su análisis y procesamiento. Se utilizó el Paquete Estadístico para las Ciencias Sociales SPSS versión 21 para el procesamiento del resto de los datos.

\section{RESULTADOS}

Esta investigación es un primer acercamiento a una serie de problemas mucho más complejos de lo que se supone con una simple mirada. Va más allá del abandono escolar y deja al descubierto problemas relacionados con la gestión escolar, el derecho a la educación y la interculturalidad, entre otros. Por razones metodológicas, se centrará la atención en los factores potenciales encontrados desde la voz de los actores educativos como respuestas para comprender el abandono escolar de los estudiantes de la Telesecundaria 531 Frida Kahlo. Se presentan en las siguientes categorías: 
a) Los usos y costumbres de los pueblos originarios

Los usos y costumbres son concebidos como las reglas particulares que rigen la política propia de los pueblos originarios, caracterizados por su forma de gobierno apegado a sus costumbres y forma de vida. En 1992 se reconocieron los derechos de los pueblos indígenas en la Constitución Política de los estados Unidos Mexicanos, en su artículo $2^{\circ}$ :

La Nación tiene una composición pluricultural sustentada originalmente en sus pueblos indígenas que son aquellos que descienden de poblaciones que habitaban en el territorio actual del país al iniciarse la colonización y que conservan sus propias instituciones sociales, económicas, culturales y políticas, o parte de ellas... Esta Constitución reconoce y garantiza el derecho de los pueblos y las comunidades indígenas a la libre determinación

Durante las entrevistas colectivas realizadas a los padres de familia durante las reuniones para conocer el abandono escolar de sus hijos, coinciden en señalar que, por usos y costumbres, las mujeres a edad temprana contraen matrimonio.

De acuerdo con la entrevista el motivo de que las alumnas de secundaria no concluyen sus estudios básicos, respondieron en un $86 \%$ que la mayor parte de alumnas en esa edad escolar contraen matrimonio de acuerdo con las tradiciones de la comunidad que se transmite de padres a hijas en la que deben de unirse a alguien para no quedarse solteras después de determinada edad.

En la entrevista un padre de familia menciona "nuestras hijas dejan de asistir a la escuela porque de acuerdo con nuestras costumbres se ocupan de los quehaceres del hogar, se casan muy jóvenes al igual que todas las mujeres de la comunidad, si alguien pide su mano, aunque esté en la escuela tiene que irse”.

Al ser entrevistados los docentes, coinciden con esta idea sobre el principal motivo de abandono, pues consideran que en un $80 \%$ las alumnas se van de la escuela por contraer matrimonio, según datos del formato de bajas de la institución.

b) Violencia de género

En las respuestas de las preguntas abiertas a padres de familia se puede apreciar que en un 78\% existe violencia de género, al manifestar que apoyan a sus hijos más que a las hijas, porque las mujeres son para el hogar y no les permiten salir a estudiar a otro lugar, las hijas tienen que sujetarse a lo que digan sus padres. Un padre de familia mencionó durante la entrevista que: "La mujer es para la 
casa, ya no damos permiso para que salga a estudiar fuera, tiene que obedecerme cuando ya no va a seguir en la escuela”.

Al ser entrevistados los docentes, en un $80 \%$ manifestaron que en cuanto a la violencia de género, las mujeres no son tomadas en cuenta en sus opiniones y no les permiten salir a realizar las tareas de investigación extraescolar.

c) La economía en el hogar

En este ciclo escolar dejaron de recibir el apoyo económico por parte del gobierno, lo que ha motivado que los alumnos dejen de asistir a la escuela y en algunos casos la abandonen. Los padres de familia al ser entrevistados sobre el apoyo a sus hijos para que continúen sus estudios académicos, en un 80 \% manifestaron que, debido a sus escasos recursos económicos y a las ocupaciones del campo, manifiestan que se ven limitados para apoyar a sus hijos en la escuela y en ocasiones los alumnos tienen que trabajar para apoyarse en sus estudios, provocando ausentismo y en algunos casos el abandono escolar. En la entrevista un padre de familia manifestó: "Como mi hijo ya no recibe la beca de prospera, tiene que ayudarme en el campo o ir a ganar con otros para comprar sus cuadernos y falta a la escuela, ya no quiere seguir estudiando porque ve que no hay dinero en la casa".

Al responder el cuestionario el $78 \%$ de los estudiantes mencionaron que no cuentan con el apoyo de sus padres para continuar sus estudios, no tienen una beca de gobierno y es probable que abandonen la escuela por falta de recursos económicos. Se perciben en una especie de encrucijada con muy pocas salidas que puedan considerarse exitosas, en comunidades con pocas oportunidades de desarrollo tanto a nivel comunitario como personal.

d) La enseñanza

Al entrevistar a los docentes coincidieron en que al $78 \%$ de los alumnos se les dificulta la comprensión de los contenidos curriculares, provocando la falta de interés en sus estudios, aunado a la baja escolaridad de los padres y la cultura que prevalece en la comunidad, lo que influye en gran medida en la deserción escolar. Un maestro de esta institución, al ser entrevistado, opina: "A los alumnos les cuesta comprender los contenidos del plan de estudios debido a su lengua materna el tzotzil, lo que propicia el desinterés en sus clases, además veo que influye la falta de apoyo de los padres 
a sus hijos, así como las costumbres arraigadas en la comunidad que se ve reflejado en el abandono escolar de los estudiantes".

De esta manera se observan las pocas oportunidades que tienen para acceder a contenidos que estén escritos en su lengua materna y que provocaría probablemente menos problemas en esa comprensión a la que aluden los profesores.

En el cuestionamiento a los alumnos de la importancia que tiene asistir a la escuela manifestaron en un $68 \%$ que es para ellos un lugar de convivencia social más que la importancia en aprender, les parece aburrido y de poca importancia terminar su educación básica, la mayoría de los estudiantes se quedan a vivir en su comunidad y se dedican a las labores del campo, en una de las preguntas abiertas del cuestionario un alumno respondió: "Me gusta la escuela porque puedo divertirme con mis amigos y puedo enamorar a mis compañeras, ya no continuaré estudiando porque tengo que apoyar a mi papá en el campo".

Nuevamente se encontró entre las respuestas del estudiantado la referente al problema económico y la forma de hacer frente a lo que les toca vivir: convivir con los otros en tanto tengan que realizar otras actividades propias de su situación en el hogar.

e) Estrategias didácticas

Al ser encuestados los docentes se puede constatar que un $90 \%$ tratan de adecuarse a los contenidos de acuerdo con el contexto de la comunidad, los alumnos son poco participativos y el avance es al ritmo de la comprensión de los estudiantes. Un docente comentó: "Los alumnos casi no participan. Tenemos que buscar estrategias para que trabajen en la clase, ellos se comunican en su lengua materna, no avanzan en sus actividades en el salón, no cumplen con las tareas, por lo que avanzamos al ritmo de la comprensión de los alumnos de acuerdo con su contexto".

Para conocer la opinión de los alumnos al contestar el cuestionario, el $85 \%$ manifestó que les parece complicada la carga de tareas y trabajos escolares, sienten pesadas las horas de clase porque algunas materias se les dificultan. Una alumna menciona: "Dejan investigaciones y el internet es lento, no tenemos dinero para impresiones, algunas tareas no le entendemos por eso nos aburrimos en la clase, matemáticas casi no le entiendo".

En los resultados se pueden apreciar dos grandes problemas como factores potenciales que pueden propiciar el abandono escolar, el primero relacionado con la economía, en una población que desde 
el punto de vista de Terigi (2014) es vulnerada y el segundo relacionado con la ausencia de una mirada intercultural para atender a las características y diversidad cultural de los estudiantes. Esto significa que abordar la problemática del abandono escolar es una tarea compleja porque como bien señalaba Espíndola y León (2002) los factores son tanto extraescolares, como en este caso los relacionados con los usos y costumbres, e intraescolares, relacionados con la falta de este enfoque intercultural, el cual ha sido resaltado por Leiva (2017).

Las poblaciones vulnerables son concebidas en una condición que afecta a vastos sectores de la población no solo urbana sino también rural, dado que están colocados en situación de vulnerabilidad y que se expresan en situaciones de desigualdad. No se puede hablar de educación con equidad cuando estas poblaciones tienen que agenciarse recursos de programas gubernamentales que como en este momento se encuentran en transición y que ha dejado de existir el programa Prospera para dar paso a las Becas Benito Juárez para jóvenes estudiantes. En el caso de esta población rural, los accesos a todos los servicios necesarios para lo educativo, como es la electricidad o el internet, son desiguales frente a otras poblaciones.

Otro aspecto importante tiene que ver con la falta de reconocimiento de su diversidad cultural, entre otras cosas relacionadas con el manejo del español como una segunda lengua a la que no han podido acceder plenamente y que limita no solo la interacción profesor-alumno sino la interacción alumnocontenido. Desde el punto de vista de Muñoz (2004, p.11) el aprendizaje intercultural "debe abarcar habilidades, actitudes y características cognitivas necesarias para tratar de manera adecuada y flexible la diversidad".

Lo anterior plantea además una problemática adicional relacionada con la formación de los docentes que se encuentran a cargo de los grupos, tal parece que el abandono es un asunto solo del estudiante, cuando las estrategias didácticas y la forma de organización del contenido también son importantes en el momento de decidir si continúa cuando les resulta difícil e incomprensible. Atender a este aspecto podría también abonar para comprender el fenómeno del abandono escolar desde la perspectiva de la formación docente. Por lo anterior, es importante destacar que estos problemas son como la punta del iceberg, señalando con ello que existen una serie de problemas que son importantes identificar para atender adecuadamente a la búsqueda de soluciones eficaces para abordar apropiadamente el abandono escolar. 


\section{CONCLUSIONES}

Los usos y costumbres sobre todo cuando se aplica a las estudiantes, las coloca en situación de desventaja atendiendo a cumplirlos y abandonando la escuela, lo cual puede implicar un reto en la investigación para identificar el impacto que esto tiene en el estudiantado. Tanto hombres como mujeres deben tomar decisiones sobre su futuro en el cumplimiento de responsabilidades que deben asumir como adultos, situación no prevista por el sistema educativo, quien los concibe como adolescentes.

Estos factores considerados de carácter interpersonal no son los únicos que se identificaron como factores potenciales para el abandono. Existen problemas de fondo que requieren un trabajo de reflexión acerca del para qué de la educación secundaria en este contexto rural indígena. Si bien la educación es un derecho, también puede haber personas que renuncien a ello por otras estrategias de sobrevivencia al ser considerados en condición de vulnerabilidad.

La investigación permitió conocer de cerca tanto los factores interpersonales como institucionales que pueden ser potencialmente detonadores para que los estudiantes abandonen la escuela sin haber concluido los cursos formales. Orozco-López (2018) señala que el cambio para que los estudiantes de pueblos originarios tengan un certificado hasta haber concluido la educación secundaria por el comunicado 047 emitido por la Secretaría de Educación Pública, provocó confusión y desánimo entre pobladores de la región Altos de Chiapas, particularmente en el municipio de Chenalhó donde narra la experiencia de educación alternativa.

Este planteamiento educativo de cursar la secundaria desde su punto de vista plantea una serie de conflictos por el alargamiento de la educación básica puesto que se tenía contemplado la educación primaria con el término de ciclo para los niños/jóvenes indígenas. Desde esta perspectiva se puede comprender el fenómeno del abandono escolar en los estudiantes de la telesecundaria donde se llevó a cabo la investigación, que si bien no es la única explicación, sí plantea problemas de fondo en lo que se refiere al derecho a la educación, sobre todo a partir de la autonomía alcanzada por estos grupos con respecto al Derecho de los pueblos indígenas por el convenio 169 de la Organización Internacional del Trabajo y los derechos de los pueblos indígenas, según acuerdo signado por la ONU.

La renuencia y renuncia a la educación secundaria desde la interculturalidad podrían explicar el peso de los usos y costumbres a tal grado que existen casos de estudiantes que no se inscriben al siguiente grado. La interculturalidad no se relaciona con el respeto por la diversidad en el aula sino va más allá en el reconocimiento de las necesidades y cosmovisiones de grupos en específico, como es el de los habitantes de pueblos originarios 
que se enfrentan a contenidos que no atienden a su realidad por estar construidos desde una visión occidentalizada.

Esta imposición de un saber occidentalizado aunque aparentemente se ha naturalizado para la gran mayoría de los estudiantes del sistema educativo mexicano, debe reconocerse que deja al descubierto una serie de retos que van desde la discriminación a la que se ven sometidos algunos estudiantes de pueblos originarios, al tener que aceptar la verdad de los textos frente a sus saberes. La discusión sobre el bilingüismo frente a la castellanización también es algo pendiente en la agenda que se debe discutir, por lo que se observó en la investigación hay una predominancia de lo segundo frente al desconocimiento o poca valorización de las lenguas de pueblos originarios.

Martínez (2016) sugiere a partir del análisis realizado a la educación indígena en el Ecuador que hace falta todavía muchas cosas por hacer a nivel estructural para hacer frente a los desafíos de una verdadera educación intercultural que recupere los saberes y las prácticas propias de cada pueblo originario sin estigmatizarlos o considerarlos fracasados, en un sistema educativo que en busca de atención a la cobertura no atiende los problemas de fondo.

Es por ello por lo que debe atenderse de manera urgente la problemática del abandono escolar desde todas sus dimensiones, para identificar cómo se está presentando en otros planteles educativos con las mismas características. Esto implica hacer una investigación más amplia que dé cuenta de la realidad de la educación telesecundaria en contextos rurales indígenas en el estado de Chiapas, lo que supone un compromiso investigativo para ser tomado en cuenta en futuros trabajos de indagación 


\section{BIBLIOGRAFÍA}

Bourdieu, P. y Passeron, J. (1081). La reproducción: elementos para una teoría del sistema de enseñanza. Laia.

Ceballos- Herrera F. (2009). El informe de investigación con estudio de casos. Magis, Revista internacional de investigación en educación, 2. 413420 https://www.redalyc.org/pdf/2810/281021548015.pdf

Consejo Nacional de Población. (2015). Datos abiertos de índice de marginación. http://www.conapo.gob.mx/es/CONAPO/Datos_Abiertos_del_Indice _de_Marginacion

Constitución Política de los Estados Unidos Mexicanos. (1992). http:// www.diputados.gob.mx/LeyesBiblio/pdf/1_240217.pdf

Diario Oficial de la Federación. (1993). Ley General de Educación, Artículo 7, Fracción IV. https://www.sep.gob.mx/work/models/sep1/Resource/ 558c2c24-ob12-4676-ad9o-8ab78086b184/ley_general_educacion.pdf

Espíndola, E. y León, A. (2002). La deserción escolar en américa latina: un tema prioritario para la agenda regional. Revista Iberoamericana de educación. 30. 39-62 https://pdfs.semanticscholar.org/49f4/d542fba211addaeoa89f4e47966389c1aef6.pdf

Estrada, M. (coord.). (2018). Abandono escolar en la educación media superior de México, políticas, actores y análisis de caso. Universidad de Guanajuato. http://www.dcsh.ugto.mx/editorial/images/abandono\%2oescolar\%20 EBOOK\%20(1).pdf

González, J. (2016). Motivación y abandono escolar en educación media. Trabajo final de grado, Facultad de Psicología. Universidad de la República de Uruguay. https://www.colibri.udelar.edu.uy/jspui/bitstream/20.500.12008/7740/1/Gonz\%C3\%A1lez\%2C\%20Jimena.pdf

González-Rodríguez, D. Vidal, J. y Vieira, M. (2016). Factores que influyen en el abandono escolar temprano. Psicología y educación: presente y futuro. Ediciones ACIPE. https://rua.ua.es/dspace/bitstream/10045/64219/1/ Psicologia-y-educacion_258.pdf

Hernández, G., Velarde A., Medina L., y Correa, G. (2019). Estrategias para disminuir el nivel deserción escolar, en el гево 40 de San Mateo del Mar. Revista educativa Educateconciencia. 22(21). 108-135. http://tecnocientifica.com.mx/educateconciencia/index.php/revistaeducate/article/ viewFile/596/654

Hernández, R., Fernández, C. y Baptista, P. (2018). Metodología de la investigación. Editorial McGraw Educación.

Ibarra, L., Fonseca, C. y Anzures, E. (2018). ¿Por qué se van de la escuela? Estudio de caso. En Estrada, M. (coord.) Abandono escolar en la educación media superior de México, políticas, actores y análisis de caso. Universidad de Guanajuato. http://repositorio.ugto.mx/bitstream/ 
20.500.12059/1357/1/abandono\%20escolar\%20EBOOK\%20\%281\%29. pdf \#page $=102$

Instituto Nacional de Estadística y Geografía (INEGI). (2015). Población https://www.inegi.org.mx/temas/estructura/

Instituto Nacional de Lenguas Indígenas (INALI). (2008). Programa de Revitalización, Fortalecimiento y Desarrollo de las Lenguas Indígenas Nacionales 2008-2012. https://www.inali.gob.mx/pdf/PINALI-2008-2012.pdf

Leiva, J. (2013). De la integración a la inclusión: Evolución y cambio en la mentalidad del alumnado universitario de educación especial en un contexto universitario español. Revista electrónica "Actualidades investigativas en educación”. 3(13). 1-27. https://www.scielo.sa.cr/scielo. php?script=sci_arttext\&pid=S1409-47032013000300025

Leiva, J. (2017) La Escuela Intercultural hoy: reflexiones y perspectivas pedagógicas. Revista Complutense de Educación, 28(1). 29-43. https:// revistas.ucm.es/index.php/RCED/article/download/48589/50121

Martínez, C. (2016). Conocimiento occidental y saberes indígenas en la educación intercultural bilingüe en el Ecuador. Alteridad. Revista de educación, 2(11) https://dspace.ups.edu.ec/bitstream/123456789/13782/1/ Alt_v11n2_Martinez.pdf

Muñoz, H. (2004) Educación escolar indígena en México: la vía oficial de la interculturalidad. Escritos. Revista del Centro de Ciencias del Lenguaje. 29. 9-49. http://cmas.siu.buap.mx/portal_pprd/work/sites/escritos/ resources/LocalContent/25/1/hectormunoz.pdf

Navarro, N. (2001). Marginación escolar en los jóvenes. Aproximación a las causas de abandono. Revista de análisis. 15, 43-50. https://books.google. com.mx/books?id=ZbzODwAAQBAJ\&pg=PA43\&lpg=PA43\&dq

Núñez, G. y Jiménez H. (2016). Evaluación de indicadores. Secretaría de Educación - Fondo de Aportaciones para la Nómina Educativa y Gasto Operativo FONE 2015. https://www.academia.edu/36923594/ Evaluaci\%C3\%B3n_de_Indicadores._Secretar\%C3\%ADa_de_ Educaci\%C3\%B3n_-

Orozco- López. (2018). ¿Autonomía educativa o interculturalidad? La educación alternativa entre los pueblos indígenas de Chiapas, México. Revista colombiana de educación, 74. 37-61. www.scielo.org.co > rcde > 0120-3916-rcde-74-00037

Sandoval, W. (2018). Factores que inciden en la deserción de los estudiantes de educación básica, secundaria y media de la institución educativa técnica Félix Tiberio Guzmán del Espinal Tolima. Tesis, Universidad de Tolima. http://45.71.7.21/bitstream/001/2897/1/T\%200945\%20768\%20 CD7005.pdf

Terigi, F. (2014). La inclusión como problema de las políticas educativas. En Feijoó, M. y Pogg, M. Educación y políticas sociales; sinergias para la inclusión. 
UNESCO. 217-234. http://www.academia.edu/download/46805833/ Educacion_y_politicas_sociales_Sinergias_para_la_inclusion.pdf\#page $=218$

Usategui, E. (1992). la sociolingüística de Basil Bernstein y sus implicaciones en el ámbito escolar. Revista de Educación, 29. http://www.educacionyfp.gob. es/dam/jcr:a25bbdaf-0693-4b85-84f7-c60545a6ac1c/re2980800486-pdf.pdf

Zúñiga, V. M. (2006). Deserción estudiantil en el nivel medio superior, causas y solución. Editorial Trillas. 